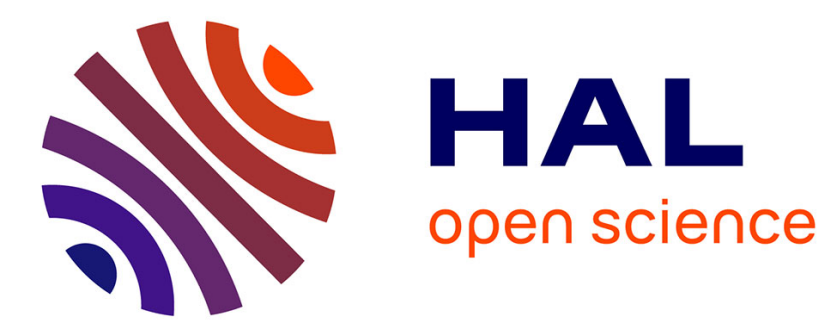

\title{
Optimization-Based Control Allocation for Driving Braking Torque Vectoring in a Race Car
}

Moad Kissai, Xavier Mouton, Bruno Monsuez, Adriana Tapus

\section{To cite this version:}

Moad Kissai, Xavier Mouton, Bruno Monsuez, Adriana Tapus. Optimization-Based Control Allocation for Driving Braking Torque Vectoring in a Race Car. American Control Conference (ACC), 2020, Denver, United States. pp.2268-2275, 10.23919/ACC45564.2020.9147343 . hal-02447091

\section{HAL Id: hal-02447091 \\ https://hal.science/hal-02447091}

Submitted on 28 Aug 2020

HAL is a multi-disciplinary open access archive for the deposit and dissemination of scientific research documents, whether they are published or not. The documents may come from teaching and research institutions in France or abroad, or from public or private research centers.
L'archive ouverte pluridisciplinaire HAL, est destinée au dépôt et à la diffusion de documents scientifiques de niveau recherche, publiés ou non, émanant des établissements d'enseignement et de recherche français ou étrangers, des laboratoires publics ou privés. 


\title{
Optimization-Based Control Allocation for Driving/Braking Torque Vectoring in a Race Car*
}

\author{
Moad Kissai $^{1}$, Bruno Monsuez ${ }^{1}$, Xavier Mouton ${ }^{2}$ and Adriana Tapus ${ }^{1}$
}

\begin{abstract}
Most of recent researches on the automotive field focus on autonomous vehicles. These vehicles are equipped with conventional chassis systems. The goal is to control the vehicle's traction, brakes, and front steering. This paper discusses the importance of advanced chassis systems, as driving/braking torque vectoring, for both autonomous and non-autonomous vehicles, especially in a race mode. Reliable co-simulation results show that expanding the vehicle's potential leads to high performances and safety with respect to severe situations when optimal control allocation is ensured. Therefore, future passenger cars shall not only be equipped with additional sensors, but also by advanced systems along with adequate control algorithms.
\end{abstract}

\section{INTRODUCTION}

The ground vehicle has always been subject to people's fascination. From the very beginning, passenger cars were considered not only as a transportation device, but also as an object of luxury and pleasure. Car manufacturers and equipment suppliers are always racing to propose new technologies in order to improve the cars' performances while keeping an acceptable level of safety. This competition is about to become fiercer with the upcoming autonomous vehicles. Not only every car manufacturer and every equipment supplier is working on this new generation of passenger cars, but even additional stakeholders are taking part in this race. This is mainly due to the fact that autonomous vehicles would require additional knowledge such as artificial intelligence, sensor fusion, cyber-security, and so on, which is not included in the know-how of the automotive industry.

One common practice to validate a new system, is to test it at its limits. In this context, researchers in [1] use simple clothoids to generate the trajectory that should be followed while respecting the friction constraints considered as the major limits [2]. Authors in [3] took over these methods to test an autonomous vehicle at the limits of handling. Results showed good performance by using an a priori knowledge of friction and a robust controller to deal with surface variations. However, these results remain far from the real potential of ground vehicles. One of the reasons of these limitations is the use of only the front steering capability of the vehicle to turn. Today's vehicles are already equipped with various

\footnotetext{
*This work was supported by the Group Renault

${ }^{1}$ Moad Kissai, Bruno Monsuez, and Adriana Tapus are with Institut Polytechnique de Paris, ENSTA Paris, Autonomous Systems and Robotics Lab, Department of Computer and System Engineering (U2IS), 828 Boulevard des Marchaux, 91120 Palaiseau, France \{moad.kissai, bruno.monsuez, adriana.tapus\}@ensta-paris.fr

${ }^{2}$ Xavier Mouton is with the Group Renault, Chassis Systems Department, 1 Avenue du Golf, 78280 Guyancourt, France xavier.mouton@renault.com
}

chassis systems and Advanced Driver Assistance Systems (ADAS). For example, the 4-Wheel Steering (4WS) system has been introduced in 1987, which makes the rear wheels steer and help the driver turn the vehicle [4]. A few years after, in 1992, the Electronic Stability Program (ESP) makes its entry [5]. This system detects an avoidance situation and use a differential braking between left and right wheels to create a yaw moment and steer back the vehicle lest its loss of control. This technology showed the relevance of making use of the four tires to handle severe scenarios as obstacle avoidance. While the ESP activates only in specific scenarios, one could think of using differential braking in more additional hazardous situations. However, using the brakes repetitively while the driver does not ask for it could be annoying and would deteriorate the tires faster. Another way to take advantage of the potential of the four tires is Torque Vectoring (TV). Here, it is the drive torque that is split between right and left wheels.

Several researches focus on the Torque Vectoring in the literature. In [6], combined yaw stability and velocity regulation are proposed by means of an electric rear axle Torque Vectoring. The controller consists of an LQR, and the drive torque is split between left and right rear wheels using a limited slip differential calculated at steady-states conditions. As long as tires are not used longitudinally and laterally at the same time, no combined slip phenomena is considered at the tire level. But in order to expand the vehicle potential, we believe that Torque Vectoring should be used also at the front tires by taking into account the combined slip for better coordination. In [7], authors tackled the problem of the four-wheel independently actuated electric vehicle. A hierarchical control architecture is adopted to enhance the vehicle stability. As a high-level controller, a Sliding Mode Control (SMC) scheme is adopted to determine the desired longitudinal and lateral forces and yaw moment. In the low-level control, an optimization algorithm is adopted to allocate the driving/braking torques to each in-wheel motor by means of the Sequential Quadratic Programming (SQP). Good performances are exhibited in few standardized scenarios. The longitudinal velocity however is not very high and do not vary in the middle of the maneuvers. Moreover, the SMC uses only the sign function for the reaching law, which causes shattering and may accelerate tire wear. In order to represent a real life situation and attract the different stakeholders, different severe scenarios should be tested.

In this paper, we follow almost the same hierarchical control architecture as the one described in [7]. In contrast, we use an $\mathcal{H}_{\infty}$ controller as the high-level controller, and 
the Weighted-Least Square (WLS) formulation with Active Set Algorithm (ASA) as a solver for the control allocation problem that distributes drive torques and also brake torques. The overall architecture is then tested by co-simulation of Matlab/Simulink ${ }^{\circledR}$ that inhabits the control logic, and Simcenter Amesim $^{\circledR}$ that contains a high-fidelity vehicle model and a reproduction in 3D of a real race road located in Magny-Cours in France. The front steering wheel angle and the speed target are generated by a Model Predictive Controller provided by Amesim ${ }^{\circledR}$ that can represents either a driver or an autopilot. The purpose of this paper is to compare a vehicle without Torque Vectoring and a vehicle with the Torque Vectoring system in a race road. We increase the speed target progressively until the loss of stability to determine the limits of each vehicle. As expected, the vehicle equipped with the TV stayed stable for higher speed values. This enabled the vehicle to complete the race faster ensuring higher performance and safety at the same time. We believe that this may facilitate future vehicles control and expand their potential by adding additional degrees of freedom, and therefore accelerate their development.

The rest of the paper is structured as follows: We start in Section II by exposing the dynamics modeling of the equipped vehicle. In Section III, the control architecture is described. The control allocation scheme is briefly detailed in Section IV. Section V presents results obtained by cosimulation of Matlab/Simulink ${ }^{\circledR}$ and Amesim ${ }^{\circledR}$. Conclusions and future works are outlined in Section VI.

\section{SYSTEM MODELING}

In [8], a review of integrated vehicle dynamics control architectures is provided. It has been shown that a multihierarchical architecture is more relevant for future overactuated vehicles for its advantages in terms of extensibility, fault-tolerance, openness, and so on. In this paper, a similar architecture is developed. In order to develop each layer apart, modeling of the vehicle's Center of Gravity (CoG) motion is separated from tire modeling.

\section{A. Vehicle's CoG Motion Modeling}

The considered vehicle is equipped with an Electric Power-Assisted Steering (EPAS) system, in-wheel motors enabling Torque Vectoring (TV) feature, and a braking-based Vehicle Dynamics Control (VDC) system that enables braking each wheel apart ${ }^{1}$. Suspensions are considered passive. Regarding the vehicle's center of gravity motions, vertical motions will be then ignored. The vertical loads are more relevant to the tire model [2]. Therefore, only a planar vehicle model is considered [9]. Nevertheless, as long as torque distribution between right and left wheels is considered, a four-wheeled vehicle model should be considered (see Fig. $\left.1^{2}\right)$.

\footnotetext{
${ }^{1}$ This system resembles therefore the ESP system, but can be activated also in different scenarios than ESP helping maintaining steering control in oversteer/understeer situations.

${ }^{2}$ The Figure shows a generalized 4-wheeled vehicle model. In our case, the vehicle is equipped with a 4-wheel steering system, but it is not activated for this study.
}

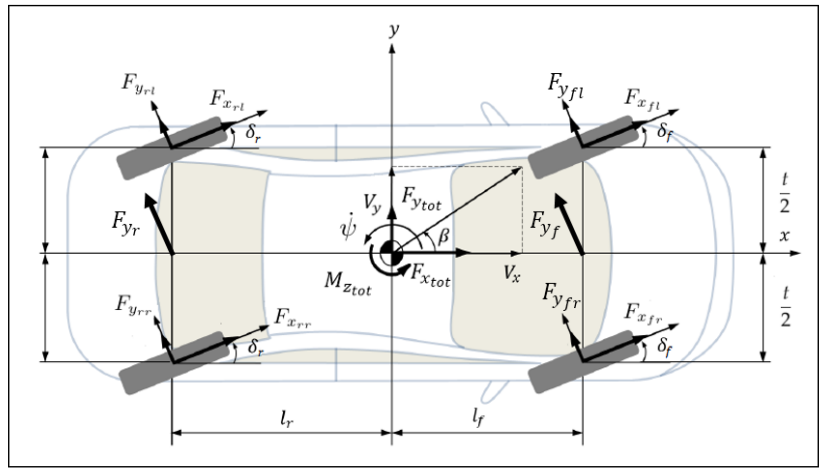

Fig. 1. The four-wheeled planar vehicle model (adapted from [9])

Using Newton's second law of motion, we can find the following state-representation [9]:

$$
\left[\begin{array}{c}
\dot{V}_{x} \\
\ddot{\psi}
\end{array}\right]=\left[\begin{array}{cc}
0 & V_{y} \\
0 & 0
\end{array}\right]\left[\begin{array}{c}
V_{x} \\
\dot{\psi}
\end{array}\right]+\left[\begin{array}{cc}
\frac{1}{M} & 0 \\
0 & \frac{1}{I_{z z}}
\end{array}\right]\left[\begin{array}{c}
F_{x_{t o t}} \\
M_{z_{t o t}}
\end{array}\right]
$$

With:

- $V_{x}$ : longitudinal velocity of the vehicle,

- $V_{y}$ : lateral velocity of the vehicle,

- $\dot{\psi}$ : yaw rate of the vehicle,

- $F_{x_{t o t}}$ : sum of longitudinal forces applied at the vehicle's Center of Gravity (CoG),

- $M_{z_{\text {tot }}}$ : sum of yaw moments applied at the vehicle's CoG,

- $M$ : vehicle's overall mass,

- $I_{z z}$ : vehicle's yaw moment of inertia.

Where:

$$
\begin{aligned}
F_{x_{t o t}}= & \left(F_{x_{f l}}+F_{x_{f r}}\right) \cos \left(\delta_{f}\right)+F_{x_{r l}}+F_{x_{r r}} \\
M_{z_{t o t}}= & \left(F_{x_{f l}}+F_{x_{f r}}\right) l_{f} \sin \left(\delta_{f}\right) \\
& +\left(F_{x_{f r}}-F_{x_{f l}}\right) \frac{t}{2} \cos \left(\delta_{f}\right)+\left(F_{x_{r r}}-F_{x_{r l}}\right) \frac{t}{2}
\end{aligned}
$$

With:

- $F_{x_{f, r}}$ : front-right longitudinal force,

- $F_{x_{f, l}}$ : front-left longitudinal force,

- $F_{x_{r, r}}$ : rear-right longitudinal force,

- $F_{x_{r, l}}$ : rear-left longitudinal force,

- $\delta_{f} \quad$ : front steering angle,

- $l_{f}$ : distance between the front axle and the CoG,

- $t \quad$ : vehicle's track.

Note that we have considered only controllable forces to distribute by the control allocation algorithm. In fact, the lateral force induced by the front steering is controlled by the MPC. Introducing this force also in the vehicle motion control induces request conflicts. As aerodynamic forces, these can be considered as disturbances to reject.

\section{B. Tire Modeling}

As front tires steers and ask for lateral tire force, a reasonable torque distribution should take into account this phenomenon to solicit more rear traction torques in case of large lateral force demand. A combined slip model is then 
needed but should remain as simple as possible to be used for control synthesis. In this paper, we use the linear tire model with varying parameters developed in [10] that fulfill these requirements. The forces are expressed as the following:

$$
\begin{aligned}
& F_{x}=C_{s}^{*}\left(\alpha, \mu, F_{z}\right) \kappa \\
& F_{y}=C_{\alpha}^{*}\left(\kappa, \mu, F_{z}\right) \alpha
\end{aligned}
$$

where:

- $\kappa$

- $\alpha$

- $\mu$

- $F_{z}$

: the longitudinal slip,

: the side-slip,

: the friction coefficient,

- $C_{s}^{*}\left(\alpha, \mu, F_{z}\right) \quad$ : the tire varying longitudinal stiffness with respect to the side-slip $\alpha, \mu$, and $F_{z}$,

- $C_{\alpha}^{*}\left(\kappa, \mu, F_{z}\right) \quad$ : the tire varying cornering stiffness with respect to the longitudinal slip $\kappa, \mu$, and $F_{z}$.

Detailed expressions of $C_{s}^{*}\left(\alpha, \mu, F_{z}\right)$ and $C_{\alpha}^{*}\left(\kappa, \mu, F_{z}\right)$ can be found in [10].

In order to respect the friction ellipse concept [2], dynamic constraints are added [10]:

$$
\begin{aligned}
& F_{x} \leq \sqrt{\left(\mu F_{z}\right)^{2}-F_{y}^{2}} \\
& F_{y} \leq \sqrt{\left(\mu F_{z}\right)^{2}-F_{x}^{2}}
\end{aligned}
$$

Here, we suppose that $\mu$ can be estimated as in [11] or [12]. Regarding the vertical load, it can be defined as in [9].

\section{CONTROL ARCHITECTURE}

As we have mentioned, today's vehicles are over-actuated. This presents additional opportunities as the vehicle's potential is extended, but presents at the same time several complexities regarding the control synthesis procedure. Typically, the torque vectoring increases the over-actuation. In [8], it is claimed that by separating the vehicle's CoG motion control from the control distribution problem, we can both simplify the control synthesis and make the overall architecture extensible to additional chassis systems and ADAS. This is of a major importance for future vehicles as their control architecture is not standardized yet, and should be therefore flexible enough. The control architecture adopted in this paper is illustrated in Fig 2.

\section{A. Control Logic Description}

In the architecture, illustrated in Fig. 2, the front steering angle $\delta_{f}$ and the speed target or speed of reference $V_{r e f}$ is generated by means of a Model Predictive Controller (MPC) provided by Amesim ${ }^{\circledR}$. Details about the MPC controller fall beyond the scope of this paper. We focus here more on the downstream control process. The MPC controller can be considered as an autopilot or a driver following a trajectory.

Once $\delta_{f}$ and $V_{\text {ref }}$ are generated, a yaw rate reference can be generated. We use the steady-state bicycle model to generate the yaw rate target [13]:

$$
\dot{\psi}_{\text {ref }}=\frac{V_{x}}{L+\frac{M V_{x}^{2}}{L C_{\alpha_{f}} C_{\alpha_{r}}}\left(C_{\alpha_{r}} l_{r}-C_{\alpha_{f}} l_{f}\right)} \delta_{f}
$$

where:

- $C_{\alpha_{f}}$ : initial cornering stiffness of front axle when no longitudinal slip is considered,

- $C_{\alpha_{r}}$ : initial cornering stiffness of rear axle when no longitudinal slip is considered,

- $l_{r} \quad$ : distance between the rear axle and the CoG,

- $L$ : vehicle's wheelbase

Due to parameters uncertainties regarding the vehicle's mass, inertia, tires' stiffness, and so on, we synthesized an $\mathcal{H}_{\infty}$ controller to ensure the robustness of the control logic. However, equation (1) shows the existence of the varying parameter $V_{y}$. This introduces a non-linearity that could be overcome by a nonlinear controller as the SMC or Gain-Scheduling. The SMC suffers from the shattering problem, which makes it unsuitable for mechanical actuators [14]. Even though this problem can be elevated by choosing adequate switch functions, the design method still lacks the flexibility and standardization that the $\mathcal{H}_{\infty}$ offers, which is essential for industrial applications. The Gain-Scheduling pass muster only for scheduling parameters that vary slowly [15]. As we aim to control a vehicle in a race mode, we have noticed fast variations of $V_{y}$ by simulations which might destabilize the vehicle dynamics. Subsequently, here we only take into account the linearized nominal model at the operating point $V_{y_{e}}=0$ to synthesize a simple $\mathcal{H}_{\infty}$ controller. This latter generates therefore the generalized commands $F_{\text {tot }}$ and $M_{t o t}$. A second controller can be added to ensure $V_{y}$ remains null. Through our investigations, we found out that this does not have considerable effects, and therefore is not justified especially since $V_{y}$ is hard to estimate in real time.

The generalized commands should be distributed into the four tires. The control allocator ensures this operation by means of optimization-based control allocation algorithms that are detailed in Section IV. The low-level control layer transforms the tire forces into torques. Equations are provided below. The different brake and drive torques commands are then sent to the Amesim ${ }^{\circledR}$ high-fidelity vehicle model to modify the vehicle behavior during the co-simulation.

\section{B. High-Level Control}

The main drawback in an $\mathcal{H}_{\infty}$ control design is the high order of the resulting controller. The order of the controller resulting is equal to the number of states in the plant plus the number of states in the requirements weights plus twice the number of states in the feedthrough matrix [16]. Here a different methodology is adopted. We add a fixedstructure of the controller as a new requirement to the control design problem. The PI structure is chosen for each controllable variable due to their integral characteristic at higher frequencies. The optimal design algorithm is operated using Matlab ${ }^{\circledR}$. In this latter, to mitigate the risk of local minima, one could run several optimizations started from randomized initial values of tunable parameters [17].

Regarding performance weighting functions, closed loop shaping is used for defining control design requirements as in [18]. Two objectives are selected: tracking performance, and commands moderation. For tracking performance, we choose 


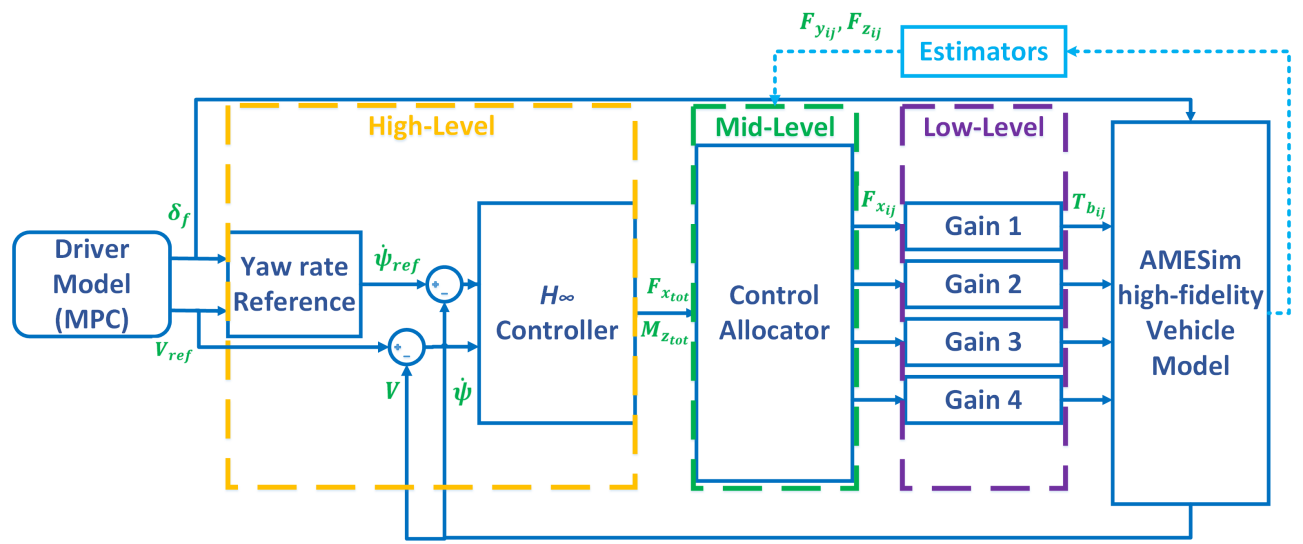

Fig. 2. The global control architecture

a steady-state offset less than $1 \%$, a closed-loop bandwidth higher than $1 \mathrm{~Hz}$, and an amplification of high-frequency noise less than a factor 3 , which gives the following weighting function:

$$
W_{\text {perf }}=\frac{1}{3} \frac{\frac{s}{2 \pi}+3}{\frac{s}{2 \pi}+0.01}
$$

With $s$ is the Laplace operator. Regarding commands moderation, we use a static gain representing the inverse of the maximum effort, which gives:

$$
W_{a c t}=\frac{1}{M g}
$$

Here, we suppose that the maximum friction coefficient is equal to 1 . The optimization algorithm gives the minimum $\mathcal{H}_{\infty}$ norm $\gamma=1.16$, which proves that the different constraints are respected and the high-level controller is stable.

\section{Low-Level Control}

The low-level control layer corresponds to the most inner loop. It should be therefore the fastest. Moreover, this layer accepts tire forces as an input. These forces are not measured in commercial sportive vehicles. For these two reasons, an open-loop control is preferred at this level. Let us note:

- $T_{b_{i, j}}$ : brake torque at the $i-j$ where $i=f$ for "front" or $r$ for "rear", and $j=l$ for "left" or $r$ for "right"wheel,

- $T_{d_{i, j}}$ : driving torque at the $i-j$ wheel,

- $R_{i}$ : wheels' effective radius of axle " $i$ ".

For simplicity, and in order to avoid adding any lag to the overall control logic, the Algorithm 1 is adopted.

Doing so, the commands reach the corresponding block at the right moment. Fig. 3 clarifies the latency at each step in the control logic.

\section{CONTROL DISTRIBUTION}

The high-level controller determines the generalized forces that should be applied at the vehicle's CoG in order to realize a specific motion. Tire forces combination should ensure the application of such forces. As long as tire forces are
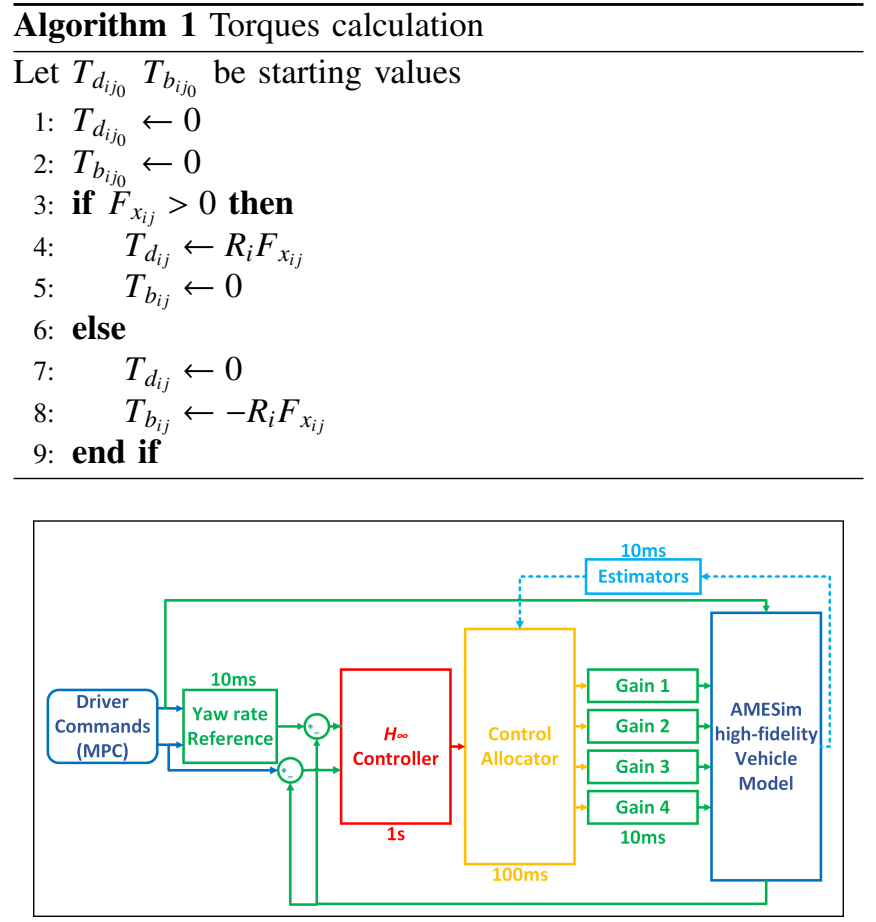

Fig. 3. Magny-Cours trajectory with the maximum performance of a vehicle without torque vectoring.

more numerous than the generalized forces, the system is considered over-actuated.

The problem has been tackled differently in the literature. Either the coordination is ensured downstream or upstream the different actuators [8]. In the downstream approach, Fuzzy logic using rule-based coordination functions has been used for example in [19]. The rules are developed with respect to some specific situations studied offline. Artificial Neural Networks have also been investigated to handle this problem downstream the systems in [20]. Here also, the network should be trained with respect to some relevant situations determined in a pre-study of vehicle dynamics. As the number of scenarios might not be scalable, the downstream approach cannot be generalized. In contrast, the upstream approach consists on formalizing the system 
interactions, describe the problem as an optimization one, and then solve it using an online optimization solver.

\section{A. Problem Definition}

The control allocation problem can be defined as follows [21]: find the control vector, $\vec{u} \in \mathbb{R}^{n}$ such that

$$
\mathbf{B}_{\text {eff }} \vec{u}=\vec{v}_{d e s}
$$

subject to

$$
\begin{aligned}
& \vec{u}_{\text {min }} \leq \vec{u} \leq \vec{u}_{\text {max }} \\
& \dot{\vec{u}} \leq \dot{\vec{u}}_{\text {max }}
\end{aligned}
$$

where $\mathbf{B}_{\text {eff }} \in \mathbb{R}^{m \times n}$ is a control effectiveness matrix, $\vec{u}_{\text {min }} \in \mathbb{R}^{n}$ and $\vec{u}_{\text {max }} \in \mathbb{R}^{n}$ are the lower and upper position limits, respectively, $\dot{\vec{u}} \in \mathbb{R}^{n}$ is the control rate, $\dot{\vec{u}}_{\text {max }} \in \mathbb{R}^{n}$ is the maximum control rate, $\vec{v}_{\text {des }} \in \mathbb{R}^{m}$ are the desired accelerations, $n$ is the number of control effectors, and $m$ is the number of axes to control.

Regarding the TV-VDC coordination case, the control vector is the following:

$$
\vec{u}=\left[\begin{array}{l}
F_{x_{f l}} \\
F_{x_{f r}} \\
F_{x_{r l}} \\
F_{x_{r r}}
\end{array}\right]
$$

$\vec{u}_{\text {min }}$ and $\vec{u}_{\text {max }}$ reflect tire limits with respect to the friction ellipse concept (6) and (7). Vertical loads variation are taken into account here using equations exposed in [22].

The desired acceleration $\vec{v}_{d e s}$ in this case is:

$$
\vec{v}_{\text {des }}=\left[\begin{array}{c}
F_{x_{t o t}} \\
M_{z_{t o t}}
\end{array}\right]
$$

The effectiveness matrix related to control allocation precision $\mathbf{B}_{\text {eff }}$ is:

$$
\mathbf{B}_{\text {eff }}=\left[\begin{array}{cccc}
\cos \left(\delta_{f}\right) & \cos \left(\delta_{f}\right) & 1 & 1 \\
b_{2,1} & b_{2,2} & -\frac{t}{2} & \frac{t}{2}
\end{array}\right]
$$

where:

$$
\begin{aligned}
\text { - } b_{2,1} & =l_{f} \sin \left(\delta_{f}\right)-\frac{t}{2} \cos \left(\delta_{f}\right), \\
\text { - } b_{2,2} & =l_{f} \sin \left(\delta_{f}\right)+\frac{t}{2} \cos \left(\delta_{f}\right) .
\end{aligned}
$$

\section{B. Problem Solving}

Several solvers can be in the literature. Sequential Least Squares (SLS) uses a two stage Active Set Algorithm (ASA) to handle two optimization problems [23]. The Weighted Least Squares (WLS) solves the bounded least squares problem using one stage ASA after few matrix transformations by means of different weights [23]. Other techniques not based on ASA can be cited as Cascading Generalized Inverses (CGI) [24] and the Fixed-Point Iteration (FPI) [25]. We choose here the WLS based on ASA for its flexibility to express multiple objectives and tune their priorities, and for its relative rapidity due to its one stage formulation. The optimal solution is then:

$$
\vec{u}_{\text {opt }}=\arg \min _{\vec{u}_{\min } \leq \vec{u} \leq \vec{u}_{\max }} \sum_{l} \gamma_{i}\left\|\mathbf{W}_{\mathbf{i}}\left(\mathbf{B}_{\mathbf{i}} \vec{u}-\vec{v}_{i}\right)\right\|^{2}
$$

- $\vec{u}_{\text {opt }} \quad$ : optimal control vector,

- $l$ : number of objectives,

- $\gamma_{i} \quad$ : weight of the $i^{\text {th }}$ objective,

- $\mathbf{W}_{\mathbf{i}}$ : non-singular weighting matrices defining preferences of each axis,

- $\overrightarrow{v_{i}} \quad$ : desired vector of the $i^{\text {th }}$ objective,

- $\mathbf{B}_{\mathbf{i}}$ : effectiveness matrix relating the control vector to the desired $i^{\text {th }}$ objective.

The solver is based on Active Set Algorithm (ASA). For details regarding this algorithm, refer to [23]. Nevertheless, it should be noted that in order to use this solver, one should rewrite the cost function following the ASA formulation:

$$
\vec{u}_{\text {opt }}=\arg \min _{\vec{u}_{\min } \leq \vec{u} \leq \vec{u}_{\max }}\|\mathbf{A} \vec{u}-\vec{b}\|
$$

So in case of a multi-objective problem, $\mathbf{A}$ and $\vec{b}$ represent vertical vectors with different specifications depending on the chosen objective. Here, to simplify, only control allocation precision is considered. No modifications are needed as the problem is naturally formulated as an ASA:

$$
\vec{u}_{\text {opt }}=\arg \min _{\vec{u}_{\min } \leq \vec{u} \leq \vec{u}_{\max }}\left\|\mathbf{W}_{\mathbf{c a}}\left(\mathbf{B}_{\text {eff }} \vec{u}-\vec{v}_{\text {des }}\right)\right\|^{2}
$$

The weighting matrix $\mathbf{W}_{\text {ca }}$ enables prioritizing the yaw rate control or the longitudinal speed control. Here, a matrix unity is considered as both controllable states are considered cardinal. For real-time maneuvers, number of iterations are limited to a finite value. A sub-optimal solution could then be generated rather than an optimal one.

\section{CO-SIMULATION RESULTS}

The selected scenario is the approved race road of the Magny-Cours area in France. This track contains bridges, hills, slopes, and the curvatures are refined to make it look like the real one. For good measure, in the MPC controller, driver parameters have been identified using a learning procedure. Dedicated maneuvers are adopted including steadystate, transient and steering feel oriented maneuvers. A nonlinear transfer function is then computed between the yaw rate and the steering wheel angle to be identified by a simple bicycle model. The computed front steering angle respects then the driver constraints regarding the steering wheel amplitude and speed.

\section{A. Simulation Setup}

The co-simulation uses the solver of Simulink ${ }^{\circledR}$ as Master and Amesim ${ }^{\circledR}$ as Slave (Fig. 4). For co-simulation in Simulink, Amesim models are exported as S-functions.

The vehicle model with its mechatronic actuators but without the control strategy is constructed in Amesim. Experimentally identified parameters of a Renault race car, as the nominal mass, inertia, tire stiffness and so on, are adopted. The aim of the presented control strategy is in fact 


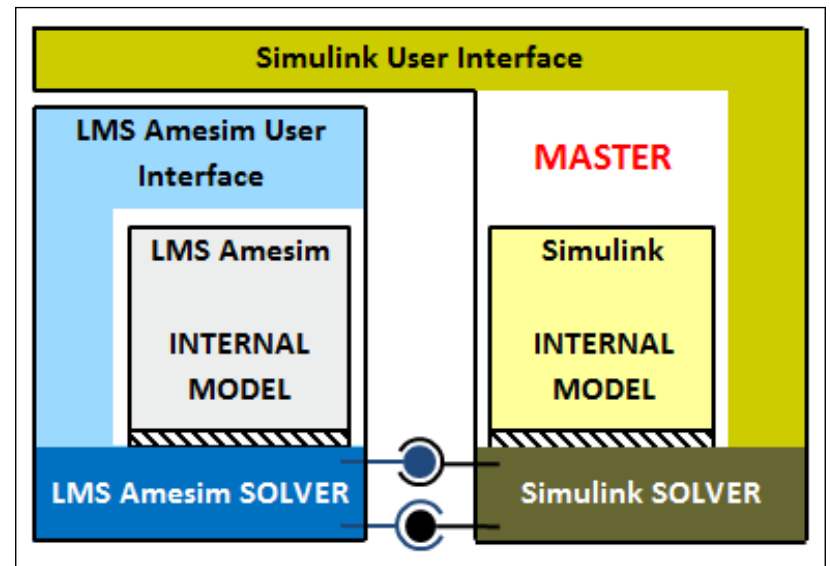

Fig. 4. Co-simulation in Simulink: Amesim as Slave, Simulink as Master.

its use in industrial applications. A 15 degrees of freedom chassis model is developed jointly with our collaborators at Renault. The vehicle model contains different interconnected modules as the suspension, antiroll bar, tire, engines, brakes, steering system and so on, all of them connected to the central module of the chassis. This model is dedicated for simultaneous longitudinal and lateral dynamics crosscouplings. The 15 degrees of freedom are due to the fact of using a multibody approach with kinematic constraints between the bodies. Complex axle kinematics are introduced to model the specific joint between sprung and unsprung masses. It should be noted that the complex kinematic tables determined empirically are provided by the car manufacturer and will depend on the type of the car. All the inertia matrix has been identified with three moments of inertia and three products of inertia. The non-controlled actuators are also well detailed. For example, even steering rack mass is modeled in Amesim. In all the following simulations, we use the carbody frame depicted in Fig. 5. Regarding the equation of motion, the basic Newton-Euler's law is applied.

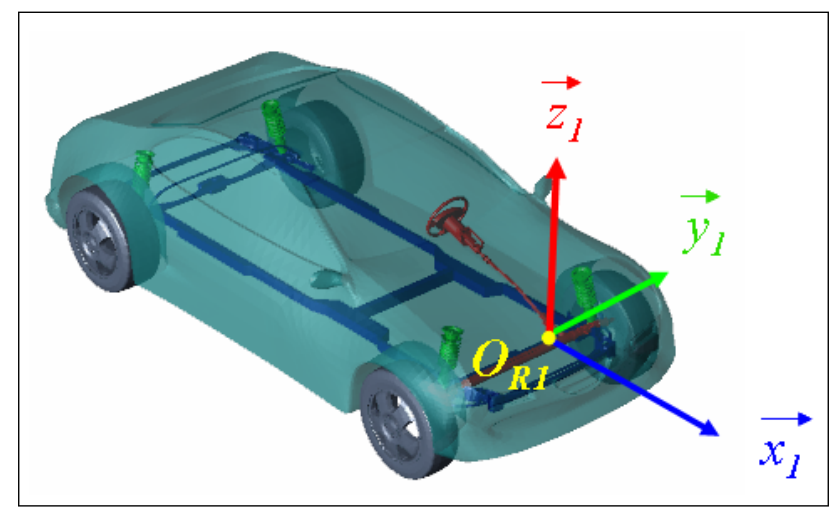

Fig. 5. Carbody frame.

The control architecture described in Section III is implemented in Simulink. The optimization-based control distribution IV is coded in Matlab. The vehicle model is called in Simulink environment as an S-function, and the whole set is co-simulated in a closed-loop using Simulink solver.
The only requirement for Windows users is the necessity of using Microsoft Visual $\mathrm{C}++$ since it is the only compiler that can generate S-Functions for Simulink. Linux users need an ANSI C Compiler that is supported by Simulink. The different PI controllers and control logics have been discretized considering a sampling time of only $10 \mathrm{~ms}$. We made all the necessary preparations to make the code work in real time. We first present the results of a vehicle not equipped with in-wheel motors and pushing it to its limits, then we provide the same maneuver but with a vehicle equipped with the Torque Vectoring system to show how much these limits can be exceeded.

\section{B. Without Torque Vectoring}

In this situation, the vehicle contains only one engine at the front axle. This engine acts equally on both front tires. Rear tires do not have any driving capabilities. Regarding the brakes, the command is only distributed between the front and rear axle by considering the static difference between the front and rear mass. As in this case the engine is located at the front axle, $60 \%$ of the brake command is allocated to the front wheels, and $40 \%$ to the rear wheels.

The maximum performance that we can get without destabilizing the vehicle is depicted in Fig. 6 .

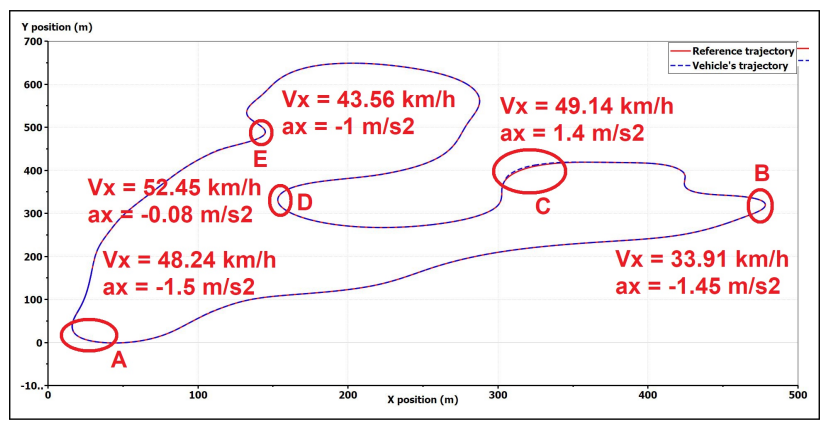

Fig. 6. Magny-Cours trajectory with the maximum performance of a vehicle without torque vectoring.

The vehicle completes the Magny-Cours trajectory in about 124.2s. Exceeding the velocity by $0.01 \mathrm{~m} / \mathrm{s}$ results on the situation illustrated in Fig 7.

The loss of control happens in Section C (see Figure 6). Among all the difficult sections, this is the only section where the vehicle was actually accelerating. In fact, the need for powertrain torque is too high for the grip capacity especially in this section. This causes front wheel spin, which leads to a global understeer behavior. This explains also the need of a steering angle value. The speed target is then reduced by the MPC, which activates the brakes. As the front wheel grip recovers, this leads to a significant load transfer that causes rear wheel blocking. As a consequence, the vehicle oversteers. Normally, a countersteering action is programmed in the MPC that consists in changing the steering wheel sign with respect to the sideslip when the latter increases too much. As this action allows the vehicle to recover its path for a maximum speed of $49.14 \mathrm{~km} / \mathrm{h}$, it remains insufficient for higher speed values. A video 


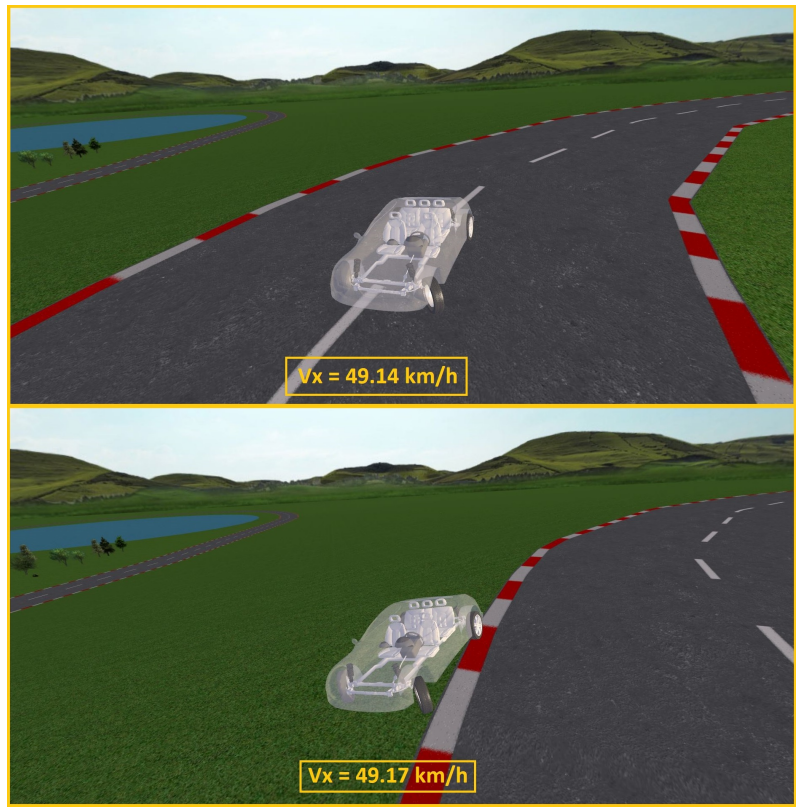

Fig. 7. Case of vehicle's loss of control when no torque vectoring is ensured.

showing the loss of control of the vehicle in this case can be visualized at the following link: Co-simulation without torque vectoring. Next, we demonstrate how an additional capability, namely the Torque Vectoring, enables exceeding this speed maximum and provides higher performances.

\section{With Torque Vectoring}

Here, the vehicle is equipped with in-wheel motors and the brakes can be controlled separately. The control architecture described in Fig. 2 is implemented in Matlab/Simulink ${ }^{\circledR}$. The generated commands are then transferred to Amesim ${ }^{\circledR}$ in a co-simulation procedure.

Fig. 8 shows the maximum speed tracking before loosing control. We can see that the vehicle has been able to enter

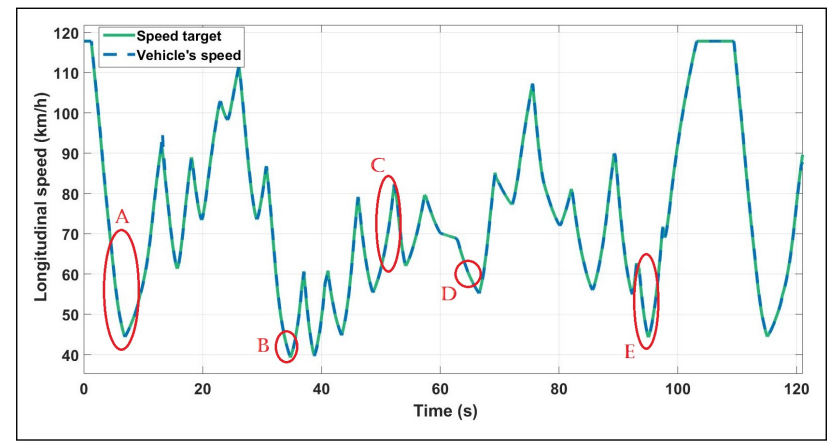

Fig. 8. Maximum speed tracking with Torque Vectoring.

the severe Section C at a speed of almost $60 \mathrm{~km} / \mathrm{h}$. In fact, the Torque Vectoring enables supplementary yaw moment than the amount provided by only the front steering. The driving-based Torque Vectoring is especially pertinent when the vehicle is accelerating, which makes it a suitable solution for the problem encountered in Section C (Fig. 9). The braking-based VDC is more relevant when the vehicle is decelerating (Fig. 10). Putting together both systems, larger values of yaw rate can be tracked (Fig. 11).

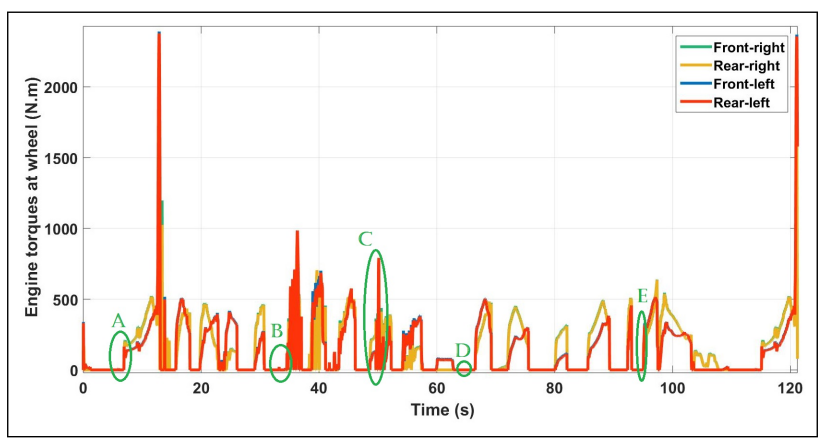

Fig. 9. Motor torques distribution at Magny-cours.

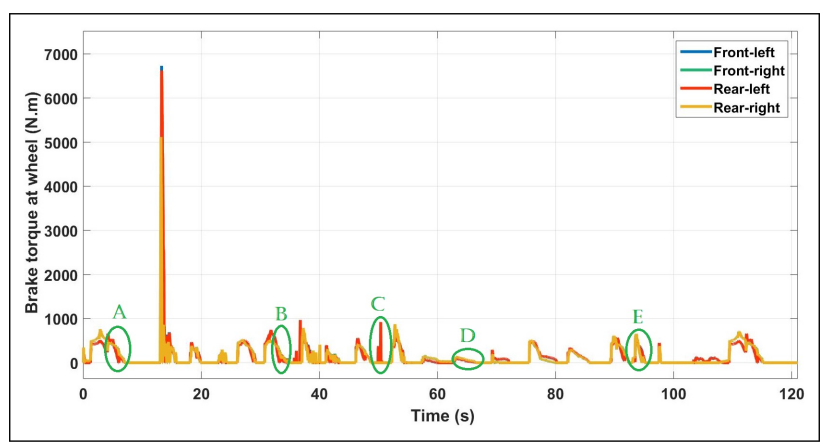

Fig. 10. Braking torques distribution at Magny-cours.

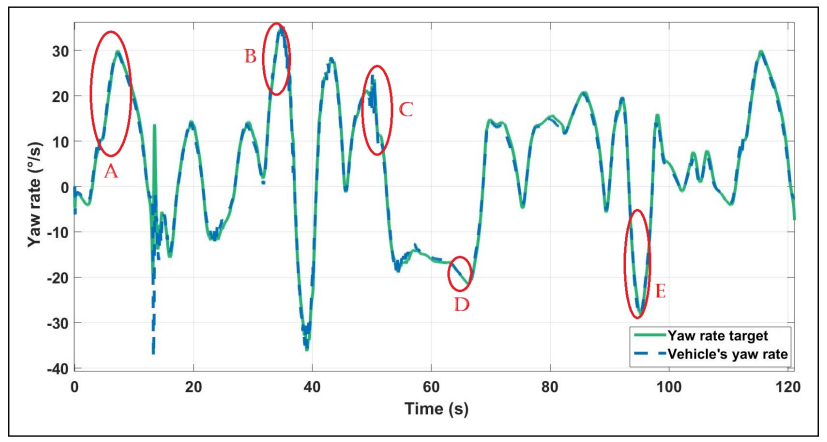

Fig. 11. Maximum yaw rate tracking with Torque Vectoring.

Thanks to this additional potential, the equipped vehicle finished the Magny-Cours circuit at only 108.2s, making a benefit of 16s. The impressive vehicle behaviour in this case, especially in Section C, can be visualized following this link: Co-simulation with torque vectoring.

Additional chassis systems with an optimal coordination give simply expanded performances. This can include realizing difficult dynamic maneuvers or complex trajectories by means of for example a 4-Wheel Steering system. Fig. 12 shows that the more numerous the chassis systems get, the more performances we can get. 


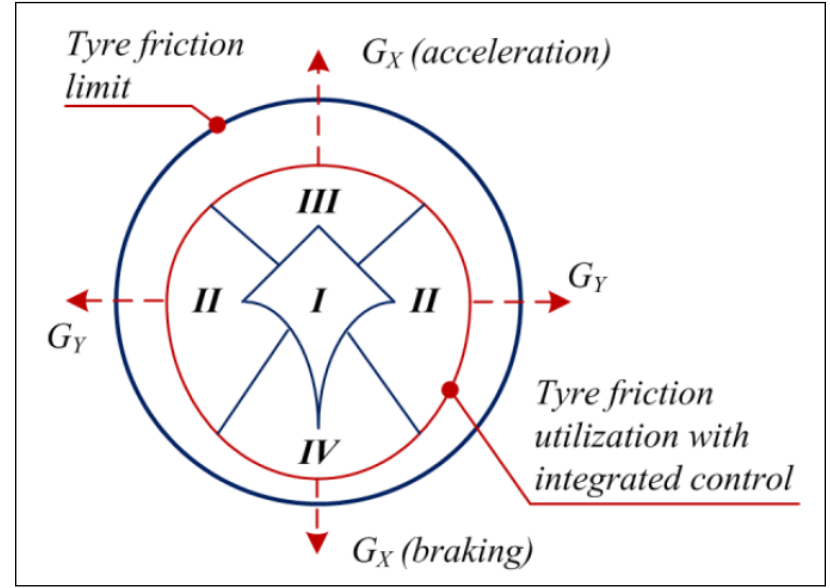

Fig. 12. Influence of integrated systems coordination on tyre utilization. Regions: I - without control; II - integration of active suspension and Active Front Steering (AFS) and ARS; III - integration of traction control and AFS and ARS; IV - integration of ABS and AFS and ARS [26].

\section{CONCLUSIONS}

In this paper, we compared two different vehicles. The first contains no Torque Vectoring features, while the second one is equipped with both a driving-based Torque Vectoring and a braking-based one. The additional chassis systems enabled the vehicle to deal with severe situations whereas the vehicle with no torque vectoring lost control at an earlier stage. Advanced chassis systems when optimally coordinated exhibit higher performances and safety. This represent additional opportunities for vehicle motion control. As we have separated trajectory tracking from dynamics control, this remains valid for both autonomous and nonautonomous vehicles. We believe that autonomous vehicles would have a big potential with an advanced intelligence, but a bigger one with additional mechatronic systems.

The authors recognize the need of experimental results. Systems' operation in real time could add few technical limitations. These systems are also very expensive. The number of systems that can be implemented within the same vehicle would be limited. It should be noted that the car manufacturer provides the vehicle with its intelligent actuators and sensors. These latter has their own physical limits. Our work is to ensure that we can benefit from the maximum potential of the car while respecting its physical limits by providing an optimal control strategy. Our ongoing work consists on experimenting optimization-based control allocation for a few set of chassis systems, and continue to test by co-simulation different chassis systems than the ones presented here to help car manufacturers adopt the right system combination. We expect at the end to provide standardizations to car manufacturers to fit their needs to a specific set of systems with an extensible vehicle dynamics control architecture.

\section{REFERENCES}

[1] J. Funke, J. Christian Gerdes, Simple Clothoid Lane Change Trajectories for Automated Vehicles Incorporating Friction Constraints, ASME. J. Dyn. Sys., Meas., Control. 2015;138(2).
[2] H.B. Pacejka, Tyre and Vehicle Dynamics, Second Edition. Elsevier, Butterworth-Heinemann, 2006.

[3] K. Kritayakirana, "Autonomous vehicle control at the limits of handling", PhD thesis, Stanford University, Stanford, CA, 2012.

[4] M. Gerard and E.Y. Lopes, "Global Chassis Control and Braking Control using Tyre Forces Measurement," TU Delft, 2011.

[5] E. Liebemann, K. Meder, J. Schuh, and G. Nenninger, "Safety and performance enhancement: The bosch Electronic Stability Control (ESP)," in SAE Technical Paper 2004-21-0060, 2004.

[6] E. Siampis, M. Massaro and E. Velenis, "Electric rear axle torque vectoring for combined yaw stability and velocity control near the limit of handling," in 52nd IEEE Conference on Decision and Control, Florence, 2013, pp. 1552-1557.

[7] Z. Wang, Y. Wang, L. Zhang, and M. Liu, "Vehicle Stability Enhancement through Hierarchical Control for a Four-Wheel-IndependentlyActuated Electric Vehicle", in Energies, vol. 10, no. 7, 2017.

[8] M. Kissai, B. Monsuez and A. Tapus, Review of integrated vehicle dynamics control architectures, 2017 European Conference on Mobile Robots (ECMR), Paris, 2017, pp. 1-8.

[9] A. Soltani, "Low Cost Integration of Electric Power-Assisted Steering (EPAS) with Enhanced Stability Program (ESP)," PhD thesis, Cranfield University, 2014.

[10] M. Kissai, B. Monsuez, A. Tapus and D. Martinez, A new linear tire model with varying parameters, 2017 2nd IEEE International Conference on Intelligent Transportation Engineering (ICITE), Singapore, 2017 , pp. 108-115.

[11] J. Svendenius, "Tire Modeling and Friction Estimation, Department of Automatic Control, Lund University, 2007.

[12] K. B. Singh and S. Taheri, "Estimation of tireroad friction coefficient and its application in chassis control systems," in Systems Science $\mathcal{E}$ Control Engineering, pp. 39-61, 2015.

[13] M. A. Selby, "Intelligent Vehicle Motion Control," PhD thesis, University of Leeds, Feb. 2003.

[14] S. Zhao, Y. Li, and X. Qu, "Vehicle Chassis Integrated Control Based on Multimodel and Multilevel Hierarchical Control," in Mathematical Problems in Engineering, vol. 2014, Article ID 248676, 2014.

[15] T. A. Johansen, I. Petersen, J. Kalkkuhl and J. Ludemann, "Gainscheduled wheel slip control in automotive brake systems," in IEEE Transactions on Control Systems Technology, vol. 11, no. 6, pp. 799811, Nov. 2003.

[16] S. Skogestad and I. Postlethwaite, Multivariable Feedback Control: Analysis and Design, 2nd Edition, JOHN WILEY \& SONS, 2001.

[17] P. Apkarian and D. Noll, "Nonsmooth H-infinity Synthesis," IEEE Transactions on Automatic Control, Vol. 51, num. 1, 2006, pp. 71-86.

[18] M. Doumiati, O. Sename, L. Dugard, J. J. Martinez Molina, P. Gaspar, et al., Integrated vehicle dynamics control via coordination of active front steering and rear braking, European Journal of Control, Lavoisier, 2013, 19 (2), pp.121-143.

[19] J.-X. Wang, N. Chen, D.-W. Pi and G.-D. Yin, "Agent-based coordination framework for integrated vehicle chassis control," in Proc. Inst. Mech. Eng., D, J. Automobile Eng., vol. 223, no. 5, pp. 601-621, 2009.

[20] C. O. Nwagboso, X. Ouyang, and C. Morgan, "Development of neuralnetwork control of steer-by-wire system for intelligent vehicles," in Heavy Vehicle Systems, vol. 9, 2002, pp. 1-26.

[21] T. A. Johansen and T. I. Fossen, Control Allocation - A survey, in Automatica, Vol. 49, Issue 5, May 2013, pp. 1087-1103.

[22] M. kissai, B. Monsuez and A. Tapus, Control Allocation of Active Rear Steering and Vehicle Dynamics Control Using a New Tire Model, International Journal of Mechanical Engineering and Robotics Research, vol. 7, no. 6, pp. 608-616, 2018.

[23] O. Harkegard, Efficient active set algorithms for solving constrained least squares problems in aircraft control allocation, Proceedings of the 41st IEEE CDC, Vol. 2, 2002, pp. 1295-1300.

[24] K.A. Bordignon, Constrained Control Allocation for Systems with Redundant Control Effectors, PhD thesis, Virginia Tech, 1996.

[25] J.J. Burken, P. Lu, Z. Wu, and C. Bahm, Two Reconfigurable FlightControl Design Methods: Robust Servomechanism and Control Allocation, Journal of Guidance, Control, and Dynamics, Vol. 24, No. 3 , pp. 482-493, 2001.

[26] V. Ivanov and D. Savitski, Systematization of Integrated Motion Control of Ground Vehicles, IEEE Access, vol. 3, pp. 2080-2099, 2015. 